\title{
Sur le renforcement des adverbes indéfinis et relatifs-interrogatifs locatifs par l'adverbe d'altérité sémantiquement apparenté en roumain contemporain
}

\author{
Adrian Chircu \\ Faculté des Lettres, Université « Babeș-Bolyai » de Cluj-Napoca, Roumanie \\ adichircu@hotmail.com
}

Dans notre intervention, à partir du corpus CoRoLa, nous nous proposons de discuter de l'adverbe d'altérité (altundeva) qui s'associe en roumain moderne à des adverbes indéfinis ou relatifs-interrogatifs locatifs, en créant des syntagmes adverbiaux complexes (oriunde altundeva, altundeva unde), à valeur de renforcement ainsi que de différenciation.

Une telle analyse peut confirmer, dans certains cas, l'appartenance du roumain à la romanité et, dans d'autres, son individualité parmi les autres langues romanes, du fait de son isolement du continuum roman et, par la suite, de sa voie évolutive particulière.

Mots-clés : roumain contemporain, CoRoLa, adverbes indéfinis, relatifs-interrogatifs et d'altérité, morphosyntaxe.

«...le groupe oriental de nos indéfinis romans diffère assez du groupe occidental par son aspect extérieur. » (Lombard 1938 : 200)

\section{Préliminaires}

Ces derniers années, nous avons pu remarquer que les linguistes s'attardent de plus en plus sur des faits de langues particuliers qui, jusqu'à présent, ont été seulement signalés sans leur accorder une attention particulière et en considérant qu'ils étaient davantage l'apanage du lexique et de la morphologie que celui de la syntaxe. En tenant compte de cette nouvelle tendance analytique, nous nous proposons de poursuivre une démarche commencée il y a trois ans (Chircu 2018), par laquelle nous avons suivi la constitution des adverbes d'altérité (ceux qui ont, dans leur structure, l'un des pronominaux latins alius ou alter) et leur diffusion en roumain et dans les différentes autres langues romanes. Ces adverbiaux ont connu un large essor et ils constituent, aujourd'hui, une classe de mots assez importante qui joue un rôle à part dans la langue populaire. 


\section{Formation des adverbes d'altérité et indéfinis}

2.1. Avant d'aborder le thème en discussion, nous estimons qu'il est nécessaire d'apporter quelques précisions concernant l'apparition de l'adverbe d'altérité (altundeva).

2.1.1. À ce propos, Mioara Avram observe que les adverbes constitués de alter + éléments constitutifs relatifs sont repérables dans d'autres langues romanes (it. altronde, altrove, engad. utró), y compris en roumain qui semble plus riche que ses consœurs en indéfinis composés (1970 : 482). Continuant l'exploit des indéfinis, cette auteure affirme que

« la richesse plus grande du roumain quant à ce type des formations ressort non seulement de leur inventaire total, mais aussi du fait que celui-ci comprend aussi bien des pronoms que des adverbes, organisés en deux séries de composés ; on constate donc que ce domaine des formations indéfinies confirme lui-aussi, la richesse particulière du roumain en ce qui concerne les composés pronominaux et adverbiaux indéfinis » (Avram 1970 : 482).

2.1.2. Généralement, on considère que l'adverbe locatif d'altérité (altundeva) est un composé, constitué de l'indéfini alter et l'adverbe indéfini undeva. Pourtant il est difficile de préciser si la particule -va, au moment de l'apparition de l'adverbe, était déjà attachée à l'indéfini (undeva), vu qu'il existe quelques attestations de cette forme composée sans la particule antérieurement mentionnée (altunde), unité lexicale qui s'est parfois conservée au niveau dialectal (Chircu 2020 : 44).

En fait, l'apparition des adverbes d'altérité se justifie par analogie avec les pronominaux indéfinis (altcine, altcineva, altce, altceva, altcare) qui étaient présents dans les premiers textes roumains et qui ont ultérieurement connu un large essor. L'association inédite de l'indéfini alter et des adverbes

«s'explique cependant aussi par la valeur nominale que prend l'adverbe dans les formations du type [...] : altunde(va) "dans un autre endroit", alaltăieri "l'autre jour" » [et aussi par] « un certain effacement de la limite entre pronoms et adverbes, un rapprochement du pronom l'autre de la classe des adverbes par sa possibilité de se combiner avec ceux-ci » (Avram 1970 : 483).

2.2. À part son achèvement successif et complexe à la fois, par l'addition à plusieurs étapes de différents éléments morphologiques, nous avons toutefois remarqué en roumain la constitution et l'épanouissement de structures syntaxiques dont les membres sont l'adverbe relatif-interrogatif (unde) ou un indéfini adverbial (oriunde) et l'adverbe d'altérité altundeva (altundeva unde / unde altundeva, oriunde altundeva / altundeva oriunde), qui sont apparus assez tardivement.

2.3. Dans les autres langues romanes, nous avons identifié des syntagmes qui, du point de vue sémantique, ont une signification semblable. Néanmoins, ceux-ci se sont réalisés différemment et leurs membres constitutifs n'ont pas la même liberté combinatoire qu'en roumain : entre autres, esp. (un) otro lugar donde, fr. un autre lieu où, port. um outro lugar onde; en roumain : un alt loc unde, mais unde în alt loc / în alt loc unde. 
2.4. L'hypothèse avancée pour leur apparition en roumain moderne se justifie par le fait que les associations syntaxiques concernées ne sont pas signalées dans les anciens textes roumains (datant des $\mathrm{XVI}^{\mathrm{e}}$ - XVIII ${ }^{\mathrm{e}}$ siècles), même si nous pouvons entrevoir de possibles modèles latins (voir, en italien, Over la mente dove altrove mira ? ${ }^{1}$ Dante, Inferno, Chant XI, vers 78, p. 110 ; aujourd'hui dove altro 'où d'autre part') qui n'ont pas réussi à s'imposer ou qui sont restés à l'état latent, caractérisant la langue populaire dont les traits dans les écrits anciens (traductions ou écrits originaux) ne sont pas toujours transparents.

\section{Repères théoriques}

Une fois délimitées la période ainsi que les modalités de constitution, nous procédons à l'analyse détaillée, en faisant appel à des faits de langue actuels qui témoigneront de la dynamique de ce type de structures en roumain contemporain qui a retenu l'attention des spécialistes, même si ceux-ci n'ont pas mené leur analyse plus loin, en se contentant seulement de la signaler.

À ce propos, dans une étude de syntaxe, Isac et Reiss (2004) s'attardent sur le syntagme générique roman dont le sens est 'something else' [« quelque chose d'autre »], en décrivant de manière générale aussi la classe des X-else qui inclut d'autres associations indéfinies complexes (something else, anything else, everywhere else, nobody else. Après avoir décrit ce type de combinaisons syntaxiques, les auteurs mentionnés rappellent la présence des structures avec des adverbiaux dans certaines langues romanes, parmi lesquelles le roumain, en lien étroit avec les pronominales. Les auteurs précisent que

«X-else expressions in Romanian always include a -va word which follows else. This -va word can either show up by itself [...], or can occur in addition to an independent $w h$-word or bare quantifier which precedes else » (Isac/Reiss 2004 : 159).

\section{Corpus d'investigation et occurrences}

4.1. À l'appui de notre étude, nous avons consulté, le 8 juillet 2019, le corpus CoRoLa qui contient un nombre significatif de textes (1 milliard de mots, 300 heures d'enregistrements, 70 sous-domaines scientifiques), où sont attestées ces structures en roumain contemporain :

(1) Mai bine începeam de oriunde altundeva. (CoRoLa)

"c'était mieux de commencer d'un tout autre endroit"

(2) nu aveam unde altundeva să o las (CoRoLa)

"je n'avais pas d'autre endroit où la laisser"

1 Isac et Reiss (2004 : 159) considèrent qu'une telle forme n'est pas repérable en italien, mais l'exemple de Dante vient infirmer cette supposition. 
(3) Unde altundeva să fii într-o duminică atât de frumoasă şi de plăcută ? (CoRoLa) "Dans quel autre endroit être un si beau et agréable dimanche?"

4.2. Après la sélection des structures concernées, nous avons réalisé une statistique qui rend compte de l'emploi et de la place de celles-ci dans la langue actuelle, en illustrant leur dynamique. Nous considérons que le nombre d'exemples est pertinent pour notre entreprise ; et la situation se présente de la manière suivante : unde altundeva (92 occurrences), oriunde altundeva (24 occurrences), altundeva unde (16 occurrences), altundeva, oriunde (1 occurrence).

\section{Interprétation des faits de langue répertoriés}

5.1. En ce qui concerne la place de l'adverbe indéfini (oriunde), nous pouvons observer que celui-ci apparaît généralement en position première, ce qui nous fait penser à une tendance au figement, avec l'alternatif en position secondaire, la postposition étant sporadique.

5.1.1. La spécificité de toutes ces constructions relativement fréquentes en roumain réside dans le double marquage sémantique de la place ( unde unde), dû au contenu sémantique du pronom d'altérité et de sa structure spécifique dont le composant principal reste l'adverbe interrogatif-relatif.

5.1.2. De telles structures, semblables au type que nous avons présenté, montrent que :

« the variable expressed by -va words duplicates the variable implicit in the wh-word or in the bare quantifier. This is exactly the type of evidence we were looking for, i.e. evidence that two NPs are involved in X-else » (Isac/ Reiss 2004 : 160).

5.2. L'élément alternatif alt- (RGR 2013 : 173), identifiable dans la structure des adverbes d'altérité, a sans doute un rôle différenciateur ; -va est, quant à lui, de provenance verbale (lat. vOLET $>$ roum. voare $>$ vare $>v a$ ) et son rôle est de marquer l'indétermination ou l'incertitude. Une implication semblable d'ordre sémantique est contenue dans la particule ori- qui se trouve dans la structure de l'adverbe indéfini (oriunde), en induisant une nuance d'indétermination ("n'importe où") (Lombard 1938: 186) et en faisant peser une nuance d'incertitude sur la réalisation de l'action.

L'association de ces deux types d'adverbes n'est pas hasardeuse, vu que les valeurs des particules de provenance verbale (<lat. vŏLĒRE) sont assez semblables. À ce propos, les auteurs de la GR observent que ces adverbes « are made up with the element oare [...] and correspond semantically to the -va» (2013:435).

5.3. Certaines structures syntaxiques repérées témoignent du fait que l'adverbe d'altérité, qui a un rôle différenciateur au sein de l'énoncé, se trouve soit antéposé (du type adverbe d'altérité + relatif-interrogatif : il est antécédent et cataphorique), soit postposé (relatif-interrogatif + adverbe d'altérité : dans ce dernier cas, il est un conséquent anaphorique, par rapport aux adverbes relatifs-interrogatifs et est employé en fonction des besoins des locuteurs). En termes d'occurrences, de loin, 
on retrouve, en position première, l'interrogatif-relatif, avec une prééminence des interrogatifs au détriment des relatifs :

(4) Săptămâna trecută, la Muzeul Satului, unde altundeva mai nimerit, s-a întâmplat o altă poveste frumoasă (CoRoLa)

“la semaine passée, au Musée du Village où, dans un autre lieu plus adéquat, il s'est passé une autre belle histoire"

(5) Prietenul meu Chris, care a venit la mine fiindcă nu are altundeva unde să se ducă (CoRoLa)

"mon ami Chris qui est venu chez moi car il n'a pas d'autre endroit où aller"

5.4. Comme nous pouvons le remarquer dans les exemples offerts, le rôle majeur de l'adverbe d'altérité est de renforcer et de doubler sémantiquement et syntaxiquement les adverbes interrogatifs-relatifs (Wh-adverbs) et indéfinis, en induisant à l'intérieur de l'énoncé, à part la nuance indéterminée, une autre différenciation qui accroît encore plus l'indétermination.

5.5. En ce qui concerne les fonctions syntaxiques des adverbes impliqués, celles-ci reflètent le contenu sémantique des adverbes relatifs-interrogatifs impliqués. Dans notre cas, il s'agit d'un circonstanciel de lieu, identifiable dans le cas de deux constituants de structures adverbiales complexes :

(6) nu aveam unde altundeva să o las (CoRoLa)

"je n'avais pas d'autre lieu où la laisser".

(7) dorindu-și cu ardoare să se afle altundeva, oriunde, dar nu acolo (CoRoLa) "en désirant ardemment se trouver dans un autre endroit, n'importe où, mais pas là"

(8) într-un memoriu care va ajunge unde altundeva decât pe biroul lui Sir David Handley Scott? (CoRoLa)

"dans un mémoire qui va arriver à un tout autre endroit que sur le bureau de Sir David Handley Scott ?"

(9) Adevărul este că nu mi s-a cerut vreodată vreo diplomă aici sau oriunde altundeva (CoRoLa)

"la vérité est qu'on ne m’a jamais demandé quelque diplôme que ce soit, ici ou ailleurs"

5.6. Dans tous ces cas, il semble que le rôle de centre de groupe adverbial (GALR II 2008 : 593 ; GR 2013 : 443) soit assumé par l'adverbe relatif-interrogatif et par l'indéfini qui contient dans sa structure la particule ori- (" free choice », RGR 2013 : 174), l'alternatif étant un adjoint qui renforce l'adverbe centre mais qui n'apporte pas de changement en ce qui concerne sa fonction grammaticale. Il ne fait que doubler la fonction syntaxique (anaphoriquement ou cataphoriquement) du centre de groupe et induire l'idée d'une éventuelle possibilité favorable pour réaliser une action. La valeur d'élément introductif (relationnel) d'une proposition circonstancielle témoigne de son rôle majeur dans l'énoncé. 
5.7. Cela confirme que les adverbes indéfinis

« sont syntaxiquement intégrés dans la proposition subordonnée, qui peut être une relative avec antécédent, situation dans laquelle l'adverbe relatif établit une relation anaphorique avec un élément de la régente quand il se substitue aussi au terme de la régente » (GALR I 2008 : 600) :

(10) În sat... după fete...unde altundeva? (CoRoLa)

"dans le village... chercher des filles...où dans un autre endroit ?"

(11) ea venise acolo fiindcă nu avusese unde altundeva să se ducă. (CoRoLa).

"elle était venue là car elle n'avait pas eu où d'autre endroit où aller"

5.8. Quant aux adverbes indéfinis à valeur relative, ceux-ci sont repérables dans « des constructions qui n'ont pas été obtenues de la transposition de la parole indirecte et sans antécédent » (GALR I 2008 : 600) mais qui, dans notre cas, apparaissent sans introduire vraiment une subordonnée :

(12) Dorim ca piața internă să funcționeze în regiunea noastră mai bine decât oriunde altundeva (CoRoLa)

"Nous désirons que le marché interne fonctionne dans notre région mieux que n'importe où ailleurs").

\section{Conclusions}

L'interprétation ponctuelle des faits de langue comme ceux que nous avons étudiés s'avère très utile et facilite une démarche appropriée visant à mieux circonscrire certaines particularités du roumain moderne et / ou contemporain.

Les concordances entre le roumain et les autres langues romanes s'expliquent sans doute par l'héritage des quelques syntagmes avec l'alternatif qui ont déjà fait leur apparition en latin vulgaire. Ensuite, durant les siècles, celles-ci ont connu un épanouissement important et un développement parfois particulier.

Malgré des évolutions sinueuses, il semble que les structures complexes interprétées restent très nombreuses dans la langue actuelle et rendent compte de la dynamique ainsi que du besoin d'apporter plus de précision au sein de l'énoncé.

\section{Bibliographie}

Aligheri, Dante (1921). La Divina Commedia [ed. Corrado Ricci], Milano : Ulrico Hoelpi.

Avram, Mioara (1970). Sur quelques pronoms et adverbes indéfinis dans les langues romanes, in : Actele celui de-al XII-lea Congres Internaţional de Lingvistică şi Filologie Romanică, vol. I, [éd. Al. Rosetti], Bucureşti : Editura Academiei Române, pp. 481-485. 
Chircu, Adrian (2020). Alteritatea adverbială dialectală românească, in : Lucrările celui de-al XVIII-lea Simpozion Internațional de Dialectologie (Cluj-Napoca, 30-31 august 2018) [éds Veronica Ana Vlasin, Rozalia Colciar, Nicolae Mocanu, Dumitru Loșonți], Cluj-Napoca : Editurile Argonaut \& Scriptor, pp. 35-58.

Chircu, Adrian (2018). La constitution de l'altérité adverbiale en roumain, in : Atti del XXVIII Congresso internazionale di linguistica e filologia romanza (Roma, 18-23 luglio 2016), vol. I, [éds Roberto Antonelli, Martin Glessgen et Paul Videsott], Strasbourg : Société de Linguistique Romane \& Éditions de Linguistique et de Philologie, pp. 658-670.

CoRoLa = Corpus computațional de referință pentru limba română contemporană, Academia Română, Bucureşti, <http://corola.racai.ro> (8/07/2019).

GALR = Guțu Romalo, Valeria (coord.) (2008). Gramatica limbii române, vol. I-II, Bucureşti : Editura Academiei Române [première édition 2005].

GR = Pană Dindelegan, Gabriela (coord.) (2013). The Grammar of Romanian, Oxford : Oxford University Press.

Isac, Daniela / Reiss Charles (2004). Romance and 'Something Else', in : Romance Languages and Linguistic Theory [eds Reineke Bok-Bennema, Bart Hollebrandse, Brigitte Kampers-Manhe et Petra Sleeman], Amsterdam / Philadelphia : John Benjamins Publishing Company, pp. 141-162.

Lombard, Alf (1938). Une classe spéciale de termes indéfinis dans les langues romanes, in : Studia Neophilologica, 9, pp. 186-209.

RGR = Dobrovie-Sorin, Carmen / Giurgea Ion (eds) (2013). A Reference Grammar of Romanian, vol. I (The noun phrase), Amsterdam-Philadelphia : John Benjamins Publishing Company.

\section{On the Reinforcement of Indefinite and Locative Relative-Interrogative Adverbs through Semantically Associated Alterity Adverb in Contemporary Romanian}

The purpose of this paper is to discuss the adverbs of alterity (altundeva "elsewhere, somewhere else") which are associated in Romanian with indefinite or relative-interrogative adverbs, thus creating complex adverbial structures (oriunde altundeva, unde altundeva "anywhere, everywhere, where else") which contain the relative-interrogative ( unde unde " where $\sim$ where") twice, with both reinforcement and differentiation function. We can add that the alterity adverb functions not only anaphorically (unde altundeva "where else") but also cataphorically (altundeva unde "elsewhere, where"), depending on the speaker's needs.

In order to make a highly complete analysis of this phenomenon, we will present common speech samples of contemporary language usage (CoRoLa Corpus).

Such an analysis can confirm, on the one hand, that the Romanian language belongs to the Roman world. However, on the other hand, it also emphasizes its individuality among other Romance languages, because of its isolation from the Roman continuum and, consequently, its particular evolution.

Key words: contemporary Romanian, CoRoLa, indefinite and relative-interrogative adverbs, alterity adverbs, morphosyntax. 
